\title{
Estrategias de afrontamiento del ciberbullying en estudiantes de secundaria pública y privada
}

\author{
Strategies to cope with cyberbullying among public and \\ private middle school students
}

DOI: https://doi.org/10.32870/dse.v0i24.1044

Josefina Sandoval Martínez*

Alejandra De Gante Casas**

María Ángela Gómez Pérez***

Rosa Margarita López Aguilar****

\section{Resumen}

El objetivo general del presente estudio fue comparar las respuestas de los estudiantes de una secundaria pública y una privada con respecto a cómo afrontarían situaciones de ciberbullying, tanto en el papel de víctimas como de testigos, para entender las respuestas de los adolescentes ante tales experiencias y aportar insumos para futuros programas de intervención que fortalezcan sus capacidades para afrontarlo eficazmente. Participaron 251 estudiantes entre 12 y 15 años, de secundarias pública y privada de la Zona Metropolitana de Guadalajara, Jalisco, durante 2019 y 2020, antes de la pandemia por Covid-19. Respondieron el Inventario de Estrategias de Afrontamiento del Cyberbullying desde la perspectiva de la Víctima (IEAC-V) y el Inventario de Estrategias de Afrontamiento del Cyberbullying desde la Perspectiva del Observador (IEAC-O). En los resultados desde la víctima, la escuela pública presentó puntuaciones elevadas en las estrategias agresivas, y en ignorar y desviar la preocupación; la escuela privada mostró puntuaciones altas en buscar apoyo, reunir pruebas y evitar al agresor. Desde el observador, la escuela pública presentó puntuaciones altas en la estrategia de ignorar y desviar la preocupación y en estrategias agresivas. Por su parte, la escuela privada presentó puntuaciones elevadas en la estrategia de reunir pruebas y buscar apoyo. Estos resultados se discuten considerando la literatura sobre ciberbullying en adolescentes.

Palabras clave: ciberbullying - estrategias de afrontamiento - víctimas - observadores.

* Doctora en Psicología. Integrante del Cuerpo Académico de Violencia, Manejo de Conflicto y Mediación. Líneas de investigación: Abordajes psicosociales de la violencia, violencia, estilos de afrontamiento ante el conflicto y mediación en el ámbito escolar, violencia y terapia familiar. Profesora-Investigadora Titular, Coordinadora de Planeación del Departamento de Psicología Aplicada, Universidad de Guadalajara. México.finasan2015@gmail.com

** Doctoranda en Psicología. Integrante del Cuerpo Académico de Violencia, Manejo de Conflicto y Mediación. Líneas de investigación: Abordajes psicosociales de la violencia, violencia, estilos de afrontamiento ante el conflicto y mediación en el ámbito escolar, violencia y terapia familiar, Profesora-Investigadora, Departamento de Psicología Aplicada, Universidad de Guadalajara. México. alejandradegantecasas@yahoo.com.mx

*** Doctora en Psicología. Responsable del Cuerpo Académico de Violencia, Manejo de Conflicto y Mediación. Líneas de investigación: Abordajes psicosociales de la violencia, violencia, estilos de afrontamiento ante el conflicto y mediación en el ámbito escolar, violencia y terapia familiar. Profesora-Investigadora, Departamento de Psicología Aplicada, Universidad de Guadalajara. México. angomez8888@hotmail.com

**** Doctora en Ciencias Sociales con especialidad en Antropología Social. Integrante del Cuerpo Académico de Violencia, Manejo de Conflicto y Mediación. Líneas de investigación: Abordajes psicosociales de la violencia, violencia, estilos de afrontamiento ante el conflicto y mediación en el ámbito escolar, violencia y terapia familiar. Profesora-Investigadora Titular, Departamento de Psicología Aplicada, Universidad de Guadalajara. México. rosilopag@gmail.com 


\begin{abstract}
The main aim of this study was to compare responses of students from a public and a private high school regarding how they would face cyberbullying situations, both as victims and as witnesses, in order to understand how adolescents respond to such experiences and provide input for future intervention programs that strengthen their ability to deal effectively with cyberbullying. 251 students aged 12 to 15 from public and private secondary schools in the Metropolitan Area of Guadalajara, Mexico participated in 2019 and 2020 before the outbreak of the COVID-19 pandemic. They answered the Inventory of Strategies for Coping with Cyberbullying from the Victim's Perspective (IEAC-V) and the Inventory of Coping Strategies for Cyberbullying from the Perspective of the Observer (IEAC-O). From the victim's perspective, the public school students had high scores in aggressive strategies as well as in ignoring and diverting concern; the private school students scored higher in seeking support, gathering evidence, and avoiding the bully. From the observer's perspective, the public school students scored higher in the strategy of ignoring and diverting concern and in aggressive strategies, while the private school students had high scores in the strategy of gathering evidence and seeking support. These results are discussed taking into account the literature on cyberbullying in adolescents.
\end{abstract}

Key words: cyberbullying - coping strategies - victims - observers.

\title{
Introducción
}

Los avances tecnológicos han venido a transformar nuestras vidas, sobre todo aquellos relacionados con las tecnologías de la información y la comunicación (TIC), que han permitido que las personas se comuniquen sin reparo del lugar geográfico en que se encuentren. La disponibilidad, accesibilidad y uso de internet ha hecho que los adolescentes desarrollen y mantengan un vínculo poderoso con éstas, situación que los ha llevado a elegirlas como la forma más frecuente y habitual para interactuar y comunicarse entre iguales de una manera rápida y efectiva.

En México, para el año 2018 había 74.3 millones de usuarios de internet de seis años o más, que representaba $65.8 \%$ de la población en ese rango de edad (INEGI, 2018), y para 2019 aumentó a 80.6 millones de usuarios, representando $70.1 \%$ de la población en el mismo rango de edad, observando un incremento de 4.3 puntos porcentuales (INEGI, 2019). Estos datos ratifican el empleo de internet, que ofrece múltiples servicios como el correo electrónico, videollamadas en línea, el uso de redes sociales como Facebook, Twitter, Instagram, etc., videoconferencias en diversas plataformas Zoom, Meet, Skype, Jitsi, entre otras, y que además fueron los medios de uso privilegiado ante la emergencia sanitaria por Covid-19, por lo que es de esperarse un aumento en los datos de usuarios de internet para 2020.

Evidentemente, este escenario ofrece una gran posibilidad de aprendizajes y formas de comunicación, pero por otra parte, también ha sido el medio para el advenimiento de comportamientos que acosan e intimidan a otros en el ciberespacio, fenómeno conocido como ciberbullying ${ }^{1}$ (Baldry, Farrington, Sorrentino, 2015).

1 En este texto, en adelante lo denominaremos ciberbuling, a manera de naturalizar el concepto en el idioma español [Nota de la correctora]. 
El ciberbuling considera aquellas prácticas agresivas que son repetitivas y con la intención de acosar e intimidar que se cometen por medio del internet por uno o varios sujetos, dirigidas a una persona (víctima) incapaz de defenderse por sí misma, evidenciando una desigualdad del poder entre el o los perpetradores y la víctima (Beltrán-Catalán, Zych, Ortega-Ruiz, Llorent, 2018; Gradinger, Yanagida, Strohmeier, Spiel, 2015; Smith, Mahdavi, Carvalho, Fisher, Russell et al., 2008). Considerando que el internet brinda la posibilidad de tener una comunicación a cualquier hora del día y desde cualquier lugar del mundo mediante un sistema de red que interconecta los dispositivos, esta situación incrementa el número de personas conectadas dando facilidades al agresor, quien puede interactuar con una identidad diferente o incluso de manera anónima para invadir, acosar y causar daño en los espacios considerados seguros por las víctimas (Herrera-López, Romera, Ortega-Ruiz, 2018; Li, 2008).

Algunos estudios a nivel mundial muestran que el ciberbuling se presenta en diversas regiones del planeta (Herrera-López, Romera, Ortega-Ruiz, 2017; Lee, Shin, 2017; Ortega, Del Rey, Casas, 2016; Waasdorp, Bradshaw, 2015); uno de cada tres menores se encuentra involucrado en alguna situación de buling tradicional y uno de cada cinco en ciberbuling (Herrera-López et al., 2018). Floridi (2007) plantea que la actual sociedad de la información soportada por las TIC tiene una gran influencia sobre las situaciones de buling tradicional (off line) que experimentan los adolescentes, y se encuentran fuertemente imbricadas con sus experiencias de ciberbuling (on line), ocasionando un fuerte impacto en la vida (on life) que permea, entre otros, el espacio educativo.

Varias investigaciones mencionan que los adolescentes participan mayormente en situaciones de ciberbuling (Beltrán-Catalán et al., 2018; Gradinger et al., 2015). Por su parte, Lucio (2014), Martínez-Vilchis, Pozas, Jiménez, Morales, Miranda et al. (2015) y Valdés Cuervo, Carlos Martínez, Tánori Quintana y Wendlandt Amezaga (2014), señalan que en México 20\% de los adolescentes intervienen como acosadores cibernéticos. Estudios sobre buling y ciberbuling en muestras mexicanas concluyen que hay una prevalencia en buling de $20 \%$ para víctimas y $19 \%$ para agresores, así como una prevalencia de ciberbuling de $21 \%$ para cibervíctimas y $11 \%$ para ciberagresión en nuestro país (Vega-Cauich, 2019).

Actualmente existe una gran preocupación social por los efectos psicológicos y sociales en niños, niñas, adolescentes y jóvenes debido a las experiencias de acoso cibernético de las que han sido objeto (León, Felipe, Fajardo, Gómez-Carroza, 2012; Matos, Vieira, Amado, Pessoa, Martins, 2018). Algunos estudios reportan que los adolescentes víctimas de ciberacoso se ven afectados con traumatismos psicoemocionales y sociales, entre otros, baja autoestima, miedo, síntomas de depresión y somatizaciones, que pueden llegar a perturbar su vida futura (Del Rey, Estévez, Ojeda, 2018; Ferrara, lanniello, Villani, Corsello, 2018; Garaigordobil, 2011; Redondo, Luzardo-Briceño, García-Lizarazo, Inglés, 2017; Schneider, O’Donnell, Stueve, Coulter, 2012). Diversos autores señalan que los resultados de tales laceraciones ocasionadas por la vivencia de 
situaciones de ciberbuling podrían ser moderadas dependiendo de la forma en que los adolescentes afronten dichas situaciones (Jacobs, Vollink, Dehue, Lechner, 2015; Machmutow, Perren, Sticca, Alsaker, 2012; Ortega-Barón, Carrascosa, 2018; Raskauskas, Huynh, 2015). Situación que explica la importancia del estudio de las estrategias de afrontamiento de los sujetos frente al ciberacoso, ya que las diferencias encontradas entre los individuos en los efectos del ciberbuling sobre el bienestar podrían deberse al uso y la efectividad de tales estrategias empleadas por ellos (Sticca, Machmutow, Stauber, Perren, Palladino et al., 2015), y cuyos resultados, favorables o no, los inclinan a percibir el acoso como estresante o aminorar dicha sensación (Torres-Acuña, Rivera, Navarro, 2019).

En su inicio, el estudio del ciberbuling se orientó a caracterizar y describir el alcance de este tipo de violencia en línea desde la perspectiva de la víctima; actualmente se incluye el estudio de los observadores de tales actos por considerar relevante su participación como testigos de tales sucesos agresivos (Machackova et al., 2017). Debido a lo anterior, el presente estudio se enfocó en analizar las estrategias de afrontamiento del ciberbuling de los adolescentes de secundaria, tanto en el rol de víctimas como de observadores o testigos de este tipo de experiencias.

\section{Estrategias de afrontamiento desde la perspectiva de la víctima}

El afrontamiento se refiere a los esfuerzos cognitivos y conductuales que los adolescentes emplean en la situación de ciberacoso, para juzgar si cuentan con los recursos necesarios para desafiarla y optar por emplear una estrategia que les ayude a resolver tal situación (Jacobs et al., 2015; Lazarus, Folkman, 1984; Mallmann, Macedo, Zanatta, 2018).

El enfoque de Lazarus y Folkman (1984), con base en un modelo transaccional, plantea una forma de afrontamiento centrado en el problema con la intención de transformar la situación que causa estrés y otra forma que se centra en la emoción, con el fin de cambiar el estado emocional del individuo. El empleo de estrategias de afrontamiento centradas en el problema en circunstancias de ciberacoso han mostrado tener mayor efectividad en sus resultados y adaptación, en comparación con el uso de las estrategias que se centran en la emoción, pues tienden a evitar la situación agresiva (Alipan, Skues, Theiler, 2018).

En este contexto, contar con un amplio conocimiento de las estrategias de afrontamiento empleadas por los adolescentes que son víctimas de acoso cibernético y continuar realizando más investigaciones (Perren et al., 2012; Raskauskas, Huynh, 2015; Espelage, Hong, 2017) favorecería el diseño de intervenciones eficaces para que, mediante éstas, los adolescentes que pudieran ser víctimas consigan fortalecer su capacidad para afrontar las consecuencias del ciberbuling (Garaigordobil, Martínez-Valderrey, 2014).

Es innegable la trascendencia del tema en cuestión para su prevención e intervención. En México, es poca la investigación sobre estrategias de afrontamiento del ciberbuling hasta el momento, no obstante, se logró identificar estudios orientados hacia estrategias de afrontamiento 
frente al buling tradicional, uno en la Ciudad de México y otro en Nuevo León. El primero con una muestra $(N=130)$ de docentes de secundarias particulares (Elizalde, 2010) y el segundo con 1,170 estudiantes adolescentes de nivel medio superior de 35 planteles educativos (Madrid, Valdés, Vera, 2015).

Al mismo tiempo, se identificaron dos estudios que, aunque no era su principal objetivo, dan cuenta de los resultados de preguntas abiertas e informan sobre las estrategias empleadas por adolescentes ante la ciberagresión (Lucio, Gómez, 2018; Vega-López, González-Pérez, Quintero-Vega, 2013).

En este aspecto, se señalan los trabajos de Torres Acuña et al. (2019) y Luna-Bernal et al. (2020) enfocados a realizar instrumentos que evalúen las estrategias de afrontamiento del ciberbuling; el primero se llevó a cabo con estudiantes universitarios, mientras que el segundo se realizó con adolescentes de bachillerato de planteles públicos del estado de Jalisco (uno regional y uno metropolitano). En sus resultados, el Inventario de Estrategias de Afrontamiento del Ciberbullying desde la perspectiva de la Víctima (IEAC-V) (Luna-Bernal et al., 2020) quedó estructurado en su versión final con siete tipos de estrategias de afrontamiento del ciberacoso desde la perspectiva de la víctima. En la tabla 1 se presenta la etiqueta y definición de cada uno de estos siete tipos de estrategias.

Tabla 1. Definiciones de estrategias de afrontamiento del ciberbuling, desde la perspectiva de la víctima

\begin{tabular}{ll}
\hline \multicolumn{1}{c}{ Estrategia } & \multicolumn{1}{c}{ Definición } \\
\hline Estrategias agresivas & $\begin{array}{l}\text { Acciones meditadas que emplea la víctima para enfrentar al aco- } \\
\text { sador de manera hostil. }\end{array}$ \\
\hline $\begin{array}{l}\text { Denunciar ante una figura de } \\
\text { autoridad }\end{array}$ & $\begin{array}{l}\text { Acciones tendientes a informar los hechos a una persona de au- } \\
\text { toridad educativa o policia, intentando que intervenga. }\end{array}$ \\
\hline $\begin{array}{l}\text { Ignorar y desviar la preocupa- } \\
\text { ción }\end{array}$ & $\begin{array}{l}\text { Esfuerzos dirigidos al autocontrol de los propios pensamientos y } \\
\text { emociones con el fin de ignorar lo que está sucediendo. }\end{array}$ \\
\hline Buscar apoyo & Búsqueda de ayuda y soporte en personas de confianza. \\
\hline Reunir pruebas & Conservar y guardar evidencias de los hechos. \\
\hline Evitar al agresor o agresores & $\begin{array}{l}\text { Utilización de medios tecnológicos y no tecnológicos para no } \\
\text { tener contacto con los agresores, sin necesidad de dejar de usar } \\
\text { internet ni lo sitios web de preferencia. }\end{array}$ \\
\hline Desconexión & $\begin{array}{l}\text { Evadir la situación evitando completamente el uso de internet o } \\
\text { de las páginas o sitios web relacionados con la situación proble- } \\
\text { mática. }\end{array}$ \\
\hline
\end{tabular}

Fuente: elaboración propia, con base en Luna-Bernal et al. (2020).

En el marco de lo anterior, el presente estudio pretende contribuir al conocimiento de los factores que podrían incidir en las preferencias de los adolescentes de secundaria por el uso de 
estas estrategias de afrontamiento del ciberbuling, comparando para ello a los estudiantes de una secundaria de sostenimiento público con una privada. No obstante, cabe mencionar a este respecto, como se señaló, que es importante para comprender la dinámica del ciberbuling desde una perspectiva psicosocial, considerar también la manera en que intervienen los observadores o testigos, tal como se explicará a continuación.

\section{Estrategias de afrontamiento del ciberbullying desde la perspectiva del observador o testigo}

Como se había mencionado anteriormente, en un principio las investigaciones sobre el ciberbuling se orientaron a analizar a la víctima; últimamente, el estudio del rol de observadores o testigos ha tomado fuerza al considerar que estos desempeñan un papel fundamental en el proceso de sucesos agresivos en línea (Machackova et al., 2017). Las reacciones de los observadores ante tales circunstancias de ciberacoso son semejantes a las del buling tradicional cuya diferencia es que suceden de manera presencial $y$, sin embargo, sus respuestas pueden igualmente afectar en el desarrollo de los hechos y sus consecuencias (Domínguez-Hernández, Bonell, Martínez-González, 2018; Elizalde, 2010).

Varios estudios con adolescentes que intervienen en situaciones de acoso señalan que los observadores presentan conductas antisociales y su manera de resolver conflictos es mediante el empleo de estrategias agresivas (Garaigordobil, 2017), además de que, debido a los comportamientos que asumen los espectadores en contextos de agresión, pueden llegar a presentar repercusiones negativas en su salud psicosocial mostrando, entre otras, sentimientos de culpabilidad, malestar, pérdida de la empatía y el desarrollo de estrés postraumático (Del Rey et al., 2018).

Ante este panorama, en el presente estudio se consideró la importancia de estudiar y generar conocimiento respecto de los adolescentes que participan como testigos u observadores y las formas que asumen para hacer frente a las situaciones de acoso en línea o ciberbuling. Múltiples estudios orientados a analizar las estrategias de afrontamiento que utilizan las personas que experimentan estas situaciones tienden a centrarse en las reacciones de las víctimas, minimizando la importancia que tienen los observadores en torno a las ciberagresiones y las estrategias que emplean (Alipan, Skues, Theiler, 2018; Jacobs, Völlink, Dehue, Lechner, 2015). Por su parte, Domínguez-Hernández et al. (2018), Knauf, Eschenbeck y Hock (2018) mencionan el interés por estudiar al observador de situaciones agresivas respecto a aquello que lo impulsa o inhibe a actuar en favor de la víctima. Recientemente, un estudio de De Gante Casas y colaboradores (2020) aborda la temática desde la perspectiva del observador, enfocándose en examinar el tipo de estrategias que emplean los adolescentes en el rol de espectadores de ciberagresiones, para lo cual diseñaron el Inventario de Estrategias de Afrontamiento del Ciberbullying desde la perspectiva del Observador (IEAC-O), con 29 reactivos agrupados finalmente 
en seis tipos de estrategias: 1) Denunciar los hechos ante una autoridad, 2) Ignorar lo que está pasando y desviar la preocupación, 3) Estrategias agresivas, 4) Reunir pruebas y buscar apoyo en una persona de confianza, 5) Evitación ansiosa o desconectarse de las redes sociales y el internet, y 6) Evitar interactuar con los agresores.

Como se puede observar, las categorías y sus definiciones son muy próximas a las expuestas en el apartado anterior. La única diferencia a este respecto es que desde la perspectiva de los observadores las estrategias de reunir pruebas y buscar apoyo en una persona de confianza quedaron reunidas en una sola categoría (a diferencia de la perspectiva de la víctima, en la que sí constituyeron dos categorías diferenciadas). Por lo demás, ambos modelos son muy semejantes. En este contexto, el presente trabajo tiene como propósito contribuir a la discusión sobre las estrategias de afrontamiento del ciberbuling desde la perspectiva de observadores o testigos de dichas situaciones.

\section{Objetivo del presente estudio}

Con base en lo anteriormente expuesto, este estudio se planteó como objetivo general comparar las respuestas de los estudiantes de una secundaria pública y una privada con respecto a cómo afrontarían situaciones de ciberbuling, tanto en el papel de víctimas como de testigos, con la finalidad de contribuir al entendimiento de los factores que podrían incidir en las maneras en que los adolescentes responden ante tales experiencias $y$, con ello, aportar un insumo para futuros programas de investigación e intervención dirigidos a fortalecer sus capacidades para un afrontamiento eficaz.

\section{Método}

\section{Participantes}

La muestra del presente estudio estuvo constituida por 251 adolescentes con rango de edad de 12 a 15 años $(M=13.27, D E=0.96)$, los cuales pertenecían a dos planteles escolares de nivel secundaria ubicados dentro de la Zona Metropolitana de Guadalajara, Jalisco, uno de sostenimiento privado y otro público. En la tabla 2 se presenta la distribución de los participantes por género, edad y grado escolar de cada una de las dos escuelas participantes.

Tabla 2. Distribución de la muestra por edad y grado escolar en función del género

\begin{tabular}{lccc}
\hline & $\begin{array}{c}\text { Escuela privada } \\
\boldsymbol{n}(\%)\end{array}$ & $\begin{array}{c}\text { Escuela pública } \\
\boldsymbol{n}(\%)\end{array}$ & $\begin{array}{c}\text { Total } \\
\boldsymbol{n}(\%)\end{array}$ \\
\hline Hombres & $62(24.9)$ & $55(22.1)$ & $117(47.0)$ \\
Mujeres & $62(24.9)$ & $70(28.1)$ & $132(53.0)$ \\
12 У 13 años & $64(25.5)$ & $81(32.3)$ & $145(57.8)$ \\
14 У 15 años & $62(24.7)$ & $44(17.5)$ & $106(42.2)$
\end{tabular}




\begin{tabular}{lccc} 
Primer grado & $41(16.3)$ & $43(17.1)$ & $84(33.5)$ \\
Segundo grado & $47(18.7)$ & $40(15.9)$ & $87(34.7)$ \\
Tercer grado & $38(15.1)$ & $42(16.7)$ & $80(31.9)$ \\
Total & $126(50.2)$ & $125(49.8)$ & $251(100.0)$ \\
\hline \multicolumn{4}{l}{ Nota. Los porcentajes de la tabla están relacionados con el total de la muestra. Hubo dos } \\
participantes que no respondieron el dato de género.
\end{tabular}

\section{Instrumentos}

Inventario de Estrategias de Afrontamiento del Ciberbullying desde la perspectiva de la Víctima (IEAC-V). Es un instrumento que se diseñó para valorar el grado en que los adolescentes tienden a emplear siete tipos de estrategias para hacer frente a posibles situaciones de ciberacoso en caso de llegar a ser víctimas: 1) Estrategias agresivas, con seis reactivos que miden las acciones meditadas empleadas por la víctima para enfrentar al acosador de manera hostil, por ejemplo: postear cosas negativas del agresor y de sus cómplices y amenazar a quienes me están acosando; 2) Denunciar, con cinco reactivos que evalúan las acciones tendientes a informar los hechos a una persona de autoridad intentando que intervenga, por ejemplo: contárselo a un profesor y contárselo al prefecto de disciplina; 3 ) Ignorar y desviar la preocupación, con seis reactivos que estiman los esfuerzos dirigidos al autocontrol de los propios pensamientos y emociones con el fin de ignorar lo que está sucediendo, por ejemplo: intentar no pensar en lo que está pasando y tomarlo con humor y pensar que no es tan grave; 4) Buscar apoyo, con cuatro reactivos que valoran la búsqueda de ayuda y soporte en personas de confianza, por ejemplo: contárselo a algún familiar y pedir ayuda a una persona de confianza y juntos pensar qué hacer al respecto; 5) Reunir pruebas, con tres reactivos que miden la conservación, y guardar evidencias de los hechos, por ejemplo: hacer captura de pantalla para guardarla como prueba; 6) Evitar al agresor, con cuatro reactivos que valoran la utilización de medios tecnológicos y no tecnológicos para no tener contacto con los agresores, sin necesidad de dejar de usar internet ni lo sitios web de preferencia, por ejemplo: evitar tener cualquier tipo de contacto con los agresores y bloquear al acosador en la cuenta (Facebook, Twitter, Instagram, etc.); y 7) Desconexión, con tres reactivos que miden el evadir la situación evitando completamente el uso de internet o de las páginas o sitios web relacionados con la situación problemática, por ejemplo: dejar de utilizar internet.

Cada reactivo se califica en una escala tipo Likert de cuatro puntos, que va desde $1=$ Definitivamente no haría eso, 2 = Probablemente no haría eso, 3 = Probablemente sí haría eso, hasta 4 = Definitivamente sí haría eso. En el presente estudio se aplicó la versión original del instrumento de 39 reactivos, pero para el análisis de datos sólo se utilizaron los 31 ítems que quedaron en su versión final, de acuerdo a la investigación realizada con 544 adolescentes estudiantes de bachillerato de dos planteles públicos ubicados en el estado de Jalisco, México. Los auto- 
res llevaron a cabo un análisis factorial exploratorio obteniendo adecuadas cargas factoriales y porcentajes de varianza explicada. Los índices de confiabilidad alfa de Cronbach reportados por estos autores fueron los siguientes: estrategias agresivas (6 reactivos) $=.86$; denunciar $(5$ reactivos) $=.83$; ignorar y desviar la preocupación ( 6 reactivos $)=.71$; buscar apoyo (4 reactivos) $=.81$; reunir pruebas $(3$ reactivos $)=.70$; evitar al agresor $(4$ reactivos $)=.72$; y desconectarse $(3$ reactivos $)=.70$. (Luna-Bernal et al., 2020).

Inventario de Estrategias de Afrontamiento del Ciberbullying desde la Perspectiva del Observador (IEAC-O). Es un instrumento que se diseñó para valorar el grado en que los adolescentes tienden a emplear seis factores relacionados con estrategias para hacer frente a posibles situaciones de ciberacoso en caso de llegar a ser espectadores o testigos. Factor 1: Denunciar, con cinco reactivos que evalúan las acciones tendientes a informar los hechos a una persona de autoridad, por ejemplo: contárselo al director de la escuela, y contárselo a alguna persona con autoridad en la escuela; Factor 2: Ignorar y desviar la preocupación, con seis reactivos que estiman comportamientos dirigidos a controlar los propios pensamientos y emociones tratando de ignorar lo que está sucediendo, por ejemplo: ignorar, hacer como que no me enteré de nada y no decirle a nadie y esperar a ver si se calma; Factor 3: Estrategias agresivas, con cinco reactivos que miden los esfuerzos cognitivos y conductuales, mediante los cuales la víctima intenta enfrentar al acosador de manera hostil, por ejemplo: insultar al agresor y a los demás que están participando o golpear al agresor; Factor 4: Reunir pruebas y buscar apoyo, con cinco reactivos que miden conservar y guardar evidencias de los hechos, y buscar ayuda y soporte, por ejemplo: no borrar las publicaciones ya que pueden servir como prueba y preguntar qué hacer a una persona de confianza; Factor 5: Desconexión, con cinco reactivos que miden rehuir la situación evitando completamente el uso de redes sociales y el internet, relacionados con la situación problemática, por ejemplo: acudir a un psicólogo y evitar entrar a todos los sitios web en que se estén dando esas agresiones; y Factor 6: Evitar al agresor, con tres reactivos que valoran utilizar medios tecnológicos y no tecnológicos para evitar interactuar con los agresores, sin necesidad de dejar de usar internet ni lo sitios web de preferencia, por ejemplo: borrar de mis contactos al agresor.

Cada reactivo se califica en una escala tipo Likert de cuatro puntos que va desde $1=$ Definitiva mente no haría eso, 2 = Probablemente no haría eso, 3 = Probablemente sí haría eso, hasta 4 = Definitivamente sí haría eso. En el presente estudio se aplicó la versión original del instrumento de 41 reactivos, pero para el análisis de datos sólo se utilizaron los 29 ítems retenidos a través de un análisis factorial exploratorio en una investigación realizada con 526 adolescentes estudiantes de bachillerato de dos planteles públicos ubicados en el Estado de Jalisco, México. El modelo explicó en total el $49.28 \%$ de la varianza con una confiabilidad alfa de Cronbach de .81 para el total de la escala. Los índices de confiabilidad alfa de Cronbach reportados por estos autores fueron de $.86, .80, .81, .82, .77$ y .68 para las escalas de denunciar, ignorar, agredir, 
guardar pruebas y buscar apoyo, desconectarse y evitar a los agresores, respectivamente (De Gante-Casas et al., 2020).

\section{Procedimiento}

Se realizaron las gestiones pertinentes a través la unidad de Orientación Educativa y de la Dirección de cada escuela, y después de plantearles los objetivos y procedimientos del estudio se obtuvo la autorización por parte de los directivos. Una vez obtenida su aprobación, se procedió a seleccionar al azar un grupo de cada grado de las escuelas secundarias para aplicar los cuestionarios durante 2019 y 2020, antes de la pandemia por Covid-19. Los grupos contestaron el instrumento en el aula, previa explicación a los alumnos acerca de los objetivos y la forma de responder los reactivos, dejando claro que no había respuestas correctas ni incorrectas, que sólo se requería que contestaran con la mayor sinceridad posible de acuerdo a sus experiencias en relación con el uso de internet, redes sociales, páginas web por ejemplo, Facebook, Twitter, Instagram, etc. También se les explicó que su participación para responder el cuestionario era voluntaria y anónima y que los resultados se manejarían únicamente con fines científicos.

\section{Resultados}

Se calcularon las puntuaciones de los participantes en cada una de las estrategias de afrontamiento del ciberbuling. Los resultados se presentan en la tabla 3. Como se puede observar, desde la perspectiva de la víctima la estrategia de buscar apoyo fue la que presentó puntuaciones más altas, seguida de reunir pruebas y evitar al agresor. Las que obtuvieron el menor puntaje fueron las estrategias agresivas. Desde el punto de vista del espectador o testigo, el mayor puntaje fue para la estrategia de reunir pruebas y buscar apoyo, seguida de evitar al agresor, mientras que el puntaje menor fue para las estrategias agresivas.

Tabla 3. Estadísticos descriptivos de las puntuaciones de los participantes en las variables de estudio

\begin{tabular}{llll}
\hline Estrategias de afrontamiento del ciberbuling & N & M & DE \\
\hline Desde la perspectiva de víctima & & & \\
Estrategias agresivas & 251 & 1.57 & 0.63 \\
Denunciar & 251 & 2.53 & 0.88 \\
Ignorar y desviar la preocupación & 251 & 2.04 & 0.62 \\
Buscar apoyo & 251 & 3.29 & 0.80 \\
Reunir pruebas & 251 & 3.28 & 0.82 \\
Evitar al agresor & 251 & 3.00 & 0.76 \\
Desconexión & 251 & 1.88 & 0.75 \\
Desde la perspectiva de observador & & &
\end{tabular}




\begin{tabular}{llll} 
Denunciar & 249 & 2.54 & 0.92 \\
Ignorar y desviar la preocupación & 249 & 1.72 & 0.63 \\
Estrategias agresivas & 249 & $\mathbf{1 . 6 0}$ & 0.60 \\
Reunir pruebas y buscar apoyo & 249 & $\mathbf{2 . 9 9}$ & 0.84 \\
Desconexión & 249 & 1.75 & 0.66 \\
Evitar al agresor & 249 & $\mathbf{2 . 7 0}$ & 0.93 \\
\hline
\end{tabular}

Fuente: elaboración propia.

Con el fin de identificar posibles diferencias estadísticamente significativas entre las respuestas de los participantes de la escuela privada y la pública, se llevó a cabo una Prueba T de Student para muestras independientes. En la tabla 4 se presentan los resultados en relación con las estrategias de afrontamiento del ciberbuling desde la perspectiva de la víctima. Como se puede observar, la escuela pública presentó puntuaciones más elevadas en las estrategias agresivas, y en ignorar y desviar la preocupación; mientras que la escuela privada mostró puntuaciones más altas en buscar apoyo, reunir pruebas y evitar al agresor.

Tabla 4. Comparativo de estrategias de afrontamiento del ciberbuling desde la perspectiva de víctima, en función del tipo de sostenimiento de la escuela

\begin{tabular}{lccc}
\hline & $\begin{array}{c}\text { Escuela privada } \\
(\boldsymbol{n}=\mathbf{1 2 6})\end{array}$ & $\begin{array}{c}\text { Escuela pública } \\
(\boldsymbol{n}=\mathbf{1 2 5})\end{array}$ & $\begin{array}{c}t \\
(\boldsymbol{g l}=\mathbf{2 4 9})\end{array}$ \\
\hline Estrategias agresivas & $1.48(0.62)$ & $\mathbf{1 . 6 6}(\mathbf{0 . 6 4})$ & $2.26 *$ \\
Denunciar & $2.57(0.80)$ & $2.45(0.95)$ & 0.83 \\
Ignorar y desviar la preocupación & $1.93(0.59)$ & $\mathbf{2 . 1 6}(0.63)$ & $2.89^{* *}$ \\
Buscar apoyo & $\mathbf{3 . 4 2 ( 0 . 7 1 )}$ & $3.15(0.87)$ & $2.80^{* *}$ \\
Reunir pruebas & $\mathbf{3 . 5 1 ( 0 . 6 2 )}$ & $3.01(0.93)$ & $4.52^{* *}$ \\
Evitar al agresor & $\mathbf{3 . 1 2 ( 0 . 6 6 )}$ & $2.87(0.83)$ & $2.52^{*}$ \\
Desconexión & $1.86(0.69)$ & $1.91(0.80)$ & 0.52 \\
\hline
\end{tabular}

Nota. $* p<.05, * * p<.01$

Por otro lado, en la tabla 5 se presentan los resultados de la Prueba T aplicada en relación con las estrategias de afrontamiento del ciberbuling desde la perspectiva de observador o testigo. Como se puede apreciar, la escuela pública presentó de manera estadísticamente significativa puntuaciones más altas en la estrategia de ignorar y desviar la preocupación y en las estrategias agresivas. Por su parte, la escuela de sostenimiento privado presentó puntuaciones más elevadas en la estrategia de reunir pruebas y buscar apoyo.

Tabla 5. Comparativo de estrategias de afrontamiento del ciberbuling desde la perspectiva de observador, en función del tipo de sostenimiento de la escuela 


\begin{tabular}{lccc}
\hline & $\begin{array}{c}\text { Escuela privada } \\
(\boldsymbol{n}=\mathbf{1 2 4})\end{array}$ & $\begin{array}{c}\text { Escuela pública } \\
(\boldsymbol{n}=\mathbf{1 2 5})\end{array}$ & $\begin{array}{c}\boldsymbol{t} \\
(\boldsymbol{g} l=\mathbf{2 4 7})\end{array}$ \\
\hline Denunciar & $2.61(0.85)$ & $2.47(0.99)$ & 1.20 \\
Ignorary desviar la preocupación & $1.63(0.60)$ & $1.82(0.65)$ & $2.40^{*}$ \\
Estrategias agresivas & $1.44(0.48)$ & $1.76(0.67)$ & $4.23^{* *}$ \\
Reunir pruebas y buscar apoyo & $3.21(0.69)$ & $2.78(0.92)$ & $4.13^{* *}$ \\
Desconexión & $1.67(0.60)$ & $1.83(0.71)$ & $2.03^{*}$ \\
Evitar al agresor & $2.72(0.88)$ & $2.67(0.97)$ & 0.42 \\
\hline
\end{tabular}

Nota. $* p<.05, * * p<.01$

\section{Discusión}

Tal como se explicó, el objetivo del presente estudio fue comparar las respuestas de los estudiantes de una secundaria pública y una privada con respecto a cómo afrontarían situaciones de ciberbuling, tanto en el papel de víctimas como de testigos. Además de ello, como un paso previo, se analizó cuáles estrategias fueron las más y menos preferidas en la muestra total de participantes.

\section{Estrategias más y menos preferidas, en general}

Como se pudo observar, desde la perspectiva de la víctima la estrategia de buscar apoyo fue la que presentó puntuaciones más altas, seguida de reunir pruebas y evitar al agresor. Las que obtuvieron el menor puntaje fueron las estrategias agresivas. Este resultado coincide con un estudio llevado a cabo por Luna-Bernal et al. (2020) con estudiantes de bachillerato, en cuanto a que el coeficiente de correlación más alto se presentó entre las estrategias de denuncia y apoyo, seguida de las correlaciones entre apoyo y pruebas, apoyo y evitación, pruebas y evitación, y denuncia y pruebas. Por su parte, las estrategias que no obtuvieron correlaciones significativas fueron las de denuncia, apoyo y desconexión, y la de pruebas con desconexión.

Desde el punto de vista del espectador o testigo, el mayor puntaje fue para la estrategia de reunir pruebas y buscar apoyo, seguida de evitar al agresor, mientras que el puntaje menor fue para las estrategias agresivas. Este resultado concuerda con el estudio de De Gante-Casas et al. (2020) con estudiantes de bachillerato, en cuanto a que el coeficiente de correlación más alto se presentó entre las estrategias de denuncia, reunir pruebas y búsqueda de apoyo, seguida de las correlaciones entre ésta y evitar al agresor. Y de manera semejante, la correlaciones que resultaron no ser estadísticamente significativa fueron las de denunciar y las estrategias agresivas.

Estos resultados pueden explicarse en función de que el sistema de educación pública de nuestro país ha venido trabajando el tema de educación para la paz en los distintos niveles escolares, la generación que ha sido objeto de este estudio ha participado a lo largo de su vida escolar en este tipo de programas; esto nos permite comprender porqué los estudiantes privi- 
legian las estrategias asertivas, congruentes con la resolución pacífica de conflictos que mostraron en ambos planteles educativos.

\section{Comparación entre una escuela pública y una privada}

Pasando al objetivo principal del presente estudio, como se pudo observar, la escuela pública presentó puntuaciones más elevadas en las estrategias agresivas, y en ignorar y desviar la preocupación; mientras que la escuela privada mostró puntuaciones más altas en buscar apoyo, reunir pruebas y evitar al agresor. Las estrategias de afrontamiento son diferentes en las escuelas públicas y privadas. En la escuela pública las estrategias son más agresivas y pueden provocar la no solución del conflicto sino su escalada a niveles más agresivos, así como el ignorar y desviar la preocupación pueden propiciar la revictimización. En el caso de la escuela privada, que favorece más buscar apoyo, reunir pruebas y evitar al agresor, propicia la denuncia, evita la confrontación y permite la intervención de otros elementos para la resolución pacífica de conflictos, evitando con ello la escalada a niveles más agresivos y la revictimización.

Finalmente, en cuanto a las estrategias de afrontamiento del ciberbuling desde la perspectiva del observador o testigo, como se pudo observar, la escuela pública presentó de manera estadísticamente significativa puntuaciones más altas en la estrategia de ignorar y desviar la preocupación y en las estrategias agresivas. Por su parte, la escuela de sostenimiento privado presentó puntuaciones más elevadas en la estrategia de reunir pruebas y buscar apoyo. Encontramos una constante en las respuestas de la víctima y de los observadores, tanto en las escuelas públicas como en las privadas: en la escuela pública se privilegia el ignorar y desviar la preocupación, además de la respuesta agresiva, como formas de resolución del conflicto, mientras que en la escuela privada privilegia el buscar apoyo y reunir pruebas para denunciar la situación problemática, inclinándose a buscar la intervención de terceros en la resolución de conflictos.

\section{Comentario final y conclusiones}

En conclusión, nos encontramos con planteles escolares desiguales en cuanto a su estructura y equipamiento, que proceden de contextos sociodemográficos diferentes que inclinan a respuestas desiguales para enfrentar el ciberbuling. Este resultado es congruente con los planteamientos propuestos por Floridi (2007) en el sentido de que las condiciones de vida off line que experimentan los adolescentes se encuentran fuertemente imbricadas con sus experiencia on line, en una sociedad de la información donde las TIC tienen un impacto on life, permeando el espacio educativo. Por lo que sería aconsejable en futuros estudios profundizar en el análisis de este fenómeno desde esta perspectiva.

Además de lo anterior, se espera que la educación sea la misma de acuerdo a lo que señala el artículo 3ro. de la Constitución; algo que no podemos negar es que la organización escolar es diferente, y por lo tanto los productos de estos procesos no son iguales. 
Desde hace más de diez años se han impulsado los programas de educación para la paz, los cuales han sido adoptados de una manera diferenciada en los centros escolares urbanos, rurales, públicos y/o privados de acuerdo a sus posibilidades. La Secretaría de Educación en el Estado de Jalisco ha establecido "Protocolos para la prevención, detección y actuación en caso de abuso sexual infantil, acoso escolar y maltrato en las escuelas de educación básica" (SEJ, s/f) con carácter de obligatorio para las escuelas públicas (DOF, 2019). Estos invitan a la denuncia y a la intervención de los directivos, docentes, estudiantes y padres de familia en la resolución pacífica de conflictos, favoreciendo las estrategias de evitación y denuncia.

Lo anteriormente descrito, y tomando en cuenta los resultados que arroja el presente trabajo, abre nuevas interrogantes para futuros estudios que nos permitan relacionar la influencia que ejercen la infraestructura, equipamiento, reglamentos escolares, cursos extracurriculares de educación para la paz, de habilidades socioemocionales, entre otros, que los alumnos reciben en los diferentes sistemas educativos, con el tipo de estrategias de afrontamiento que utilizan como víctima o como observador. Con todo lo anterior, el presente estudio espera contribuir al entendimiento de los factores que podrían incidir en las maneras en que los adolescentes responden ante las experiencias de ciberbuling $y$, con ello, aportar un insumo para futuros programas de investigación e intervención dirigidos a fortalecer sus capacidades para un afrontamiento asertivo, eficaz y pacífico.

\section{Referencias}

Alipan, A.; J. Skues; S. Theiler (2018). "They Will Find Another Way to Hurt You": Emerging Adults' Perceptions of Coping with Cyberbullying. Emerging Adulthood, 1-13. https://doi. org/10.1177/2167696818816896

Baldry, A.; D. Farrington; A. Sorrentino (2015). Am I at Risk of Cyberbullying? A Narrative Review and Conceptual Framework for Research on Risk of Cyberbullying and Cybervictimization: The Risk and Needs Assessment Aproach. Aggression and Violent Behavior, 23, 36-51. https:// doi.org/10.1016/j.avb.2015.05.014

Beltrán-Catalán, M.; I. Zych; R. Ortega-Ruiz; V. Llorent (2018). Victimisation through Bullying and Cyberbullying: Emotional Intelligence, Severity of Victimization and Technology Use in Different Types of Victims. Psicothema, 30(2), 183-188. https://doi.org/10.7334/psicothe$\underline{\text { ma2017.313 }}$

De Gante-Casas, A.; A. Luna-Bernal; R. López-Aguilar; J. Nava-Preciado (2020). Diseño y estudio psicométrico de un inventario para evaluar estrategias de afrontamiento del ciberbullying desde la perspectiva del observador. Revista Educación y Desarrollo, 52, 37-48.

Del Rey, R.; M. Estévez; M. Ojeda (2018). El ciberacoso y su respuesta educativa. En Jiménez, E.; M. Garmendia; M. Casado (coords.). Entre selfies y whatsapps. Oportunidades y riesgos para la infancia y la adolescencia conectada. Barcelona: Gedisa, 125-139. 
Diario Oficial de la Federación (2019). Ley General de Educación. Diario Oficial de la Federación. Artículo 12, fracción IV. México: DOF. http://www.diputados.gob.mx/LeyesBiblio/ref/lge.htm Domínguez-Hernández, F.; L. Bonell: A. Martínez-González (2018). A Systematic Literature Review of Factors that Moderate Bystanders' Actions in Cyberbullying. Cyberpsychology: Journal of Psychosocial Research on Cyberspace, 12(4), article 1. https://doi.org/10.5817/CP20184-1

Elizalde, A. (2010). Estudio descriptivo de las estrategias de afrontamiento del bullying en profesorado mexicano. Electronic Journal of Research in Educational Psychology, 8(1), 353-372.

Espelage, D.; J. Hong (2017). Cyberbullying Prevention and Intervention Efforts: Current Knowledge and Future Directions. The Canadian Journal of Psychiatry, 62(6), 374-380. https://doi.org/10.1177/0706743716684793

Ferrara, P.; F. Ianniello; A. Villani; G. Corsello (2018). Cyberbullying a Modern form of Bullying: Let's Talk about this Health and Social Problem. Italian Journal of Pediatrics, 44(1), 14. https://doi. org/10.1186/s13052-018-0446-4

Floridi, L. (2007). Una mirada al impacto futuro de las TIC en nuestras vidas. La Sociedad de la Información, 23(1), 59-64. https://doi.org/10.1080/01972240601059094

Garaigordobil, M. (2011). Prevalencia y consecuencias del ciberbullying: una revisión. International Journal of Psychology and Psychological Therapy, 11(2), 233-254.

Garaigordobil, M.; V. Martínez-Valderrey (2014). Cyberprogram 2.0. Programa de intervención para prevenir y reducir el ciberbullying. Madrid: Ediciones Pirámide.

Garaigordobil, M. (2017). Conducta antisocial: Conexión con bullying/cyberbullying y estrategias de resolución de conflictos [Antisocial Behavior: Connection with Bullying/Cyberbullying and conflict resolution]. Psychosocial Intervention, 26(1), 47-54. https://doi.org/10.1016/j. psi.2015.12.002

Gradinger, P.; T. Yanagida; D. Strohmeier; C. Spiel (2015). Prevention of Cyberbullying and Cybervictimization: Evaluation of the ViSC Social Competence Program. Journal of School Violence, 14(1), 87-110. https://doi.org/10.1080/15388220.2014.963231

Herrera-López, M.; E. Romera; R. Ortega-Ruiz (2017). Bullying y cyberbullying en Colombia; coocurrencia en adolescentes escolarizados. Revista Latinoamericana de Psicología, 49(3), 163172. https://doi.org/10.1016/j.rlp.2016.08.001

Herrera-López, M.; E. Romera; R. Ortega-Ruiz (2018). Bullying y cyberbullying en Latinoamérica: un estudio bibliométrico. Revista Mexicana de Investigación Educativa, 23(76), 125-155.

Instituto Nacional de Estadística y Geografía (2018). Encuesta Nacional sobre Disponibilidad y Uso de Tecnologías de la Información en los Hogares (ENDUTIH). (Comunicado de prensa). México: INEGI. https://www.inegi.org.mx/contenidos/saladeprensa/boletines/2019/OtrTemEcon/ ENDUTIH 2018.pdf 
Instituto Nacional de Estadística y Geografía (2019). Encuesta Nacional sobre Disponibilidad y Uso de Tecnologías de la Información en los Hogares (ENDUTIH). (Comunicado de prensa). México: INEGI. http://www.ift.org.mx/sites/default/files/comunicacion-y-medios/comunicados-ift/ comunicadoendutih $0 . p d f$

Jacobs, N.; T. Vollink; F. Dehue; L. Lechner (2015). The Development of a Self-Report Questionnaire on Coping with Cyberbullying: The Cyberbullying Coping Questionnaire. European Societies, 5(2), 460-491. https://doi.org/10.3390/soc5020460

Knauf, R.; H. Eschenbeck; M. Hock (2018). Bystanders of Bullying: Social-Cognitive and Affective Reactions to School Bullying and Cyberbullying. Cyberpsychology: Journal of Psychosocial Research on Cyberspace, 12(4), article 3. http://dx.doi.org/10.5817/CP2018-4-3

Lazarus, R.; S. Folkman (1984). Stress, Appraisal, and Coping. Nueva York: Springer Publishing Company.

Lee, C.; N. Shin (2017). Prevalence of Cyberbullying and Predictors of Cyberbullying Perpetration among Korean Adolescents. Computers in Human Behavior, 68, 352-358. https://doi. org/10.1016/j.chb.2016.11.047

Lucio, L. (2014). Cyberbullying en alumnos del nivel medio superior. Tesis de Licenciatura. México: Universidad Autónoma del Estado de México. Repositorio Institucional. http://hdl.handle. net/20.500.11799/49383

Lucio, L.; F. Gómez (2018). Las redes sociales como campo de batalla, el cyberbullying en estudiantes del Nivel Medio Superior. En Sánchez-Castañeda, A. (coord.). Acoso escolar y cyberbullying. Retos, prevención y sensibilización. México: Universidad Nacional Autónoma de México, Defensoría de los Derechos Universitarios, 105-115.

Luna-Bernal, A.; M. Gómez-Pérez; J. Sandoval-Martínez; A. Valencia-Aguirre (2020). Estructura factorial y confiabilidad de un inventario para evaluar estrategias de afrontamiento del cyberbullying desde la perspectiva de la víctima. Revista Educación y Desarrollo, (53), 71-81.

Machackova, H.; J. Pfetsch; G. Steffgen (2017). Editorial: Special Issue on Bystanders of Online Aggression. Cyberpsychology: Journal of Psychosocial Research on Cyberspace, 12(4). https:// doi.org/10.5817/CP2018-4-xx

Machmutow, K.; S. Perren; F. Sticca; F. Alsaker (2012). Peer Victimisation and Depressive Symptoms: Can Specific Coping Strategies Buffer the Negative Impact of Cybervictimisation? Emotional and Behavioural Difficulties, 17(3-4), 403-420. https://doi.org/10.1080/13632752 .2012 .704310

Madrid, E.; A. Valdéz; J. Vera (2015). Diferencias en los estilos de afrontamiento de estudiantes víctimas y no involucrados en el bullying. XIII Congreso Nacional de Investigación Educativa. Chihuahua, México. http://www.comie.org.mx/congreso/memoriaelectronica/v13/ doc/0133.pdf 
Mallmann, C.; C. Macedo; T. Zanatta (2018). Cyberbullying and Coping Strategies in Adolescents from Southern Brazil. Acta Colombiana de Psicología, 21(1), 13-43. https://doi.org/10.14718/ ACP.2018.21.1.2

Martínez-Vilchis, R.; J. Pozas; K. Jiménez; M. Morales; D. Miranda; M. Delgado; V. Cuenca (2015). Prevención de la violencia cara a cara y virtual en el bachillerato. Psychology, Society y Education, 7(2), 201-212.

Matos, A.; C. Vieira; J. Amado; T. Pessoa; M. Martins (2018). Cyberbullying in Portuguese Schools: Prevalence and Characteristics. Journal of School Violence, 17(1), 123-137. https://doi.org/1 $\underline{0.1080 / 15388220.2016 .1263796}$

Ortega, R.; R. Del Rey; J. Casas (2016). Evaluar el bullying y el cyberbullying validación española del EBIP-Q. Psicología Educativa, 22(1), 71-79. https://doi.org/10.1016/j.pse.2016.01.004

Perren, S.; L. Corcoran; H. Cowie; F. Dehue; D. Garcia; C. Mc Guckin; D. Smahel; A. Sevcikova; P. Tsatsou; T. Vollink (2012). Coping with Cyberbullying: A Systematic Literature review. Final Report of the COST IS0801 Working Group 5, Paris. París: International Conference on Cyberbullying. http://kops.uni-konstanz.de/handle/123456789/23800

Raskauskas, J.; A. Huynh (2015). The Process of Coping with Cyberbullying: A Systematic Review. Aggression and Violent Behavior, 23, 118-125. https://doi.org/10.1016/j.avb.2015.05.019

Redondo, J.; M. Luzardo-Briceño; K. García-Lizarazo; C. Inglés (2017). Impacto psicológico del ciberbullying en estudiantes universitarios: un estudio exploratorio. Revista Colombiana de Ciencias Sociales, 8(2), 458-478. https://doi.org/10.21501/22161201.2061

Secretaría de Educación Jalisco (s/f). Protocolos para la prevención, detección y actuación en caso de abuso sexual infantil, acoso escolar y maltrato en las escuelas de educación básica. México: SEJ. http://edu.jalisco.gob.mx/programa-nacional-convivencia-escolar/sites/edu.jalisco. gob.mx programa-nacional-convivencia-escolar/files/protocolos casos de abuso sexual infantil acoso escolar y maltrato en las escuelas.pdf

Schneider, S.; L. O'Donnell; A. Stueve; R. Coulter (2012). Cyberbullying, School Bullying, and Psychological Distress: A Regional Census of High School Students. American Journal of Public Health, 102(1), 171-177. https://doi.org/10.2105/AJPH.2011.300308

Smith, P.; J. Mahdavi; M. Carvalho; S. Fisher; S. Russell; N. Tippett (2008). Cyberbullying: its Nature and Impact in Secondary School Pupils. Journal of Child Psychology and Psychiatry, 49(4), 376-385.

Sticca, F.; K. Machmutow; A. Stauber; S. Perren; B. Palladino; A. Nocentini; E. Menesini; L. Corcoran; C. Mc Guckin (2015). The Coping with Cyberbullying Questionnaire: Development of a New Measure. Societies, 5(2), 515-536. https://doi.org/10.3390/soc5020515

Torres-Acuña, G.; C. Rivera; Y. Navarro (2019). Validación de una escala para medir afrontamiento ante ciberagresiones entre universitarios. Revista Electrónica de Investigación Educativa, 21(e09), 1-11. https://doi.org/10.24320/redie.2019.21.e09.1907 
Valdés, A.; E. Carlos; J. Tánori; T. Wendlandt (2014). Differences in Types and Technological Means by which Mexican High Schools Students Perform Cyberbullying: Its Relationship with Traditional Bullying. Journal of Educational and Developmental Psychology, 4(1), 105-113. http:// dx.doi.org/10.5539/jedp.v4n1p105

Vega-Cauich, J. (2019). Prevalencia del bullying en México: un meta-análisis del bullying tradicional y cyberbullying. Diversitas, 15(1), 111-127. https://doi.org/10.15332/22563067.4020

Vega, M.; G. González-Pérez; P. Quintero-Vega (2013). Ciberacoso: victimización de alumnos en escuelas secundarias públicas de Tlaquepaque, Jalisco, México. Educación y Desarrollo, 25, 13-20.

Waasdorp, T.; C. Bradshaw (2015). The Overlap between Cyberbullying and Traditional Bullying. Journal of Adolescent Health, 56(5), 483-488. https://doi.org/10.1016/j. jadohealth.2014.12.002 